University of Wollongong

Research Online

Australian Institute for Innovative Materials -

Papers

Australian Institute for Innovative Materials

$1-1-2016$

\title{
Higher order plasmonic modes excited in Ag triangular nanoplates by an electron beam
}

Vicki J. Keast

University of Newcastle

C J. Walhout

University of Twente

T Pedersen

University of Newcastle

N Shahcheraghi

University of Technology Sydney

Michael Cortie

University of Technology Sydney, michael.cortie@uts.edu.au

See next page for additional authors

Follow this and additional works at: https://ro.uow.edu.au/aiimpapers

Part of the Engineering Commons, and the Physical Sciences and Mathematics Commons

Research Online is the open access institutional repository for the University of Wollongong. For further information contact the UOW Library: research-pubs@uow.edu.au 


\title{
Higher order plasmonic modes excited in Ag triangular nanoplates by an electron beam
}

\author{
Abstract \\ Ag triangular nanoplates are known to generate strong plasmonic resonances when excited by both light \\ and electron beams. Experimental electron energy-loss spectra (EELS) and maps were acquired using an \\ aberration-corrected JEOL-ARM microscope. The corner, edge and centre modes that are often observed \\ in such structures were also observed in these measurements. In addition, novel higher order internal \\ modes were observed and were found to be well reproduced by theoretical cal- culations using boundary \\ element method (BEM). These modes are "dark modes" so are not observed in the optical extinction \\ spectra. They are confined surface propagating modes and are analogous to laser cavity modes

\section{Keywords} \\ excited, modes, plasmonic, order, higher, beam, electron, nanoplates, triangular, ag \\ Disciplines \\ Engineering | Physical Sciences and Mathematics

\section{Publication Details} \\ Keast, V. J., Walhout, C. J., Pedersen, T., Shahcheraghi, N., Cortie, M. B. \& Mitchell, D. R. G. (2016). Higher \\ order plasmonic modes excited in Ag triangular nanoplates by an electron beam. Plasmonics, 11 (4), \\ 1081-1086.
}

\section{Authors}

Vicki J. Keast, C J. Walhout, T Pedersen, N Shahcheraghi, Michael Cortie, and David R. G Mitchell 
Higher order plasmonic modes excited in Ag triangular nanoplates by an electron beam

\author{
V. J. Keast ${ }^{1 *}$, C. J. Walhout ${ }^{2}$, T. Pedersen ${ }^{1}$, N. Shahcheraghi ${ }^{3}$, M. B. Cortie ${ }^{3}$, D. R. G. Mitchell ${ }^{4}$ \\ ${ }^{1}$ School of Mathematical and Physical Sciences, University of Newcastle, Callaghan NSW 2308, \\ Australia. \\ ${ }^{2}$ University of Twente, 7500 AE Enschede, The Netherlands. \\ ${ }^{3}$ Institute for Nanoscale Technology, University of Technology Sydney. PO Box 123, Broadway, \\ NSW 2007, Australia \\ ${ }^{4}$ Electron Microscopy Centre (EMC), Australian Institute for Innovative Materials (AIIM), \\ Innovation Campus, University of Wollongong, NSW 2500, Australia.
}

${ }^{*}$ Corresponding author.

Assoc. Prof. Vicki J. Keast

School of Mathematical and Physical Sciences

The University of Newcastle

Callaghan NSW 2308

Australia

Ph: + 61249216653

Fax: + 61249216907

email: vicki.keast@newcastle.edu.au

\begin{abstract}
Ag triangular nanoplates are known to generate strong plasmonic resonances when excited by both light and electron beams. Experimental electron energy-loss spectra (EELS) and maps were acquired using an aberration corrected JEOL-ARM microscope. The corner, edge and centre modes that are often observed in such structures were also observed in these measurements. In addition, novel higher order internal modes were observed and were found to be well-reproduced by theoretical calculations using boundary element method (BEM). These modes are "dark modes" so are not observed in the optical extinction spectra. They are confined surface propagating modes and are analogous to laser cavity modes.
\end{abstract}

\title{
Keywords
}

silver nanoparticles, localized surface plasmon resonance, electron energy-loss spectroscopy (EELS), boundary element method (BEM)

\section{Acknowledgements}

This research was supported under Australian Research Council's Discovery Projects funding scheme (Project Number DP120102545) and used equipment funded by the Australian Research Council (ARC) - Linkage, Infrastructure, Equipment and Facilities (LIEF) grant LE120100104 located at the UOW Electron Microscopy Centre. Masashi Watanabe is thanked for providing his PCA code. Interpretation of the spectral features was assisted by use of the ACEOS code, written by Thomas A. Myles. 


\section{Introduction}

Metal nanoparticles of a variety of different shapes and compositions have been investigated in recent years for plasmonic applications [1-6]. A material which stands out due to its strong response throughout the visible and near-infrared regions is Ag. One particular structure under study is the triangular nanoplate, where the frequency of the plasmon response can be readily modified with edge length, aspect ratio and tip truncation [7-12]. Along with a relatively straightforward wet chemical synthesis route [13], these features make triangular Ag nanoplates attractive for the study and application of plasmonics.

Localised surface plasmon resonances (LSPRs) in nanoparticles can be excited via both light and electrons, but electron beam excitation is less widely used. The optical characteristics of triangular nanoplates have been investigated by many photonic spectroscopic measurements where most involve averaging over a population of particles in a solution or on a substrate [7-9,14-21], however, it has also been possible to record the optical response of individual particles [10,22-24].

The extinction spectra of triangular Ag nanoplates are dominated by a long-wavelength peak at around 700-800 $\mathrm{nm}$ and further smaller peaks at shorter wavelengths. The long-wavelength peak has been assigned as the in-plane dipole peak, and the other peaks can, depending on direction of electric field and shape of the particle, be assigned as higher order in-plane peaks (quadrupole, octapole and decapole) or out-of-plane dipole and quadrupole excitations [21,25,26]. In-plane and out-of-plane refers to LSPRs excited by different polarization directions of the incident light relative to the plane of the triangular plate. Size, shape effects, the dielectric environment and coupling effects between two or more particles have all been observed to alter the plasmon response. For triangular nanoplates the aspect ratio has been found to have a strong effect on the inplane dipole peak while the higher order peaks show little sensitivity to this $[9,10,12,24,25]$.

The limits in spatial resolution when using optical excitation of LSPRs can be overcome by using the sub-nanometre electron beam available in the electron microscope, in conjunction with electron energy-loss spectroscopy (EELS). Using EELS, not only can the plasmon responses from individual nanoparticles be measured, but a map of resonances excited at different electron beam positions within a nanoparticle can be generated, revealing important connections between geometry and plasmon resonances. There have been several EELS investigations of triangular nanoplates which have revealed the presence of resonances that occur when the electron beam is located at the corners the edges and in the centre of the nanoprism [11,27-33] and these resonances have been correlated to peaks found in the optical extinction spectra using theoretical simulations. The corner mode corresponds to the dipole resonance, the beam at middle of the edge mode excites a quadrupole resonance while a beam positioned at approximately one-quarter of the edge excites a octupolar resonance. The centre mode is not excited optically and is a so-called dark mode.

This work presents EELS measurements of the plasmon modes in large Ag triangular nanoplates using an aberration corrected, cold-field-emission-gun (CFEG) scanning transmission electron microscope (STEM). In addition to the modes excited at the corners and edge of the nanoplate, a number of higher order internal modes are observed for the first time. These observations are supported by calculations using the boundary element method (BEM) [34-36].

\section{Methods}

Samples of Ag triangular nanoplates were prepared by reacting $\mathrm{AgNO}_{3}$ and polyvinylpyrrolidone (the latter as a protective surfactant) with $\mathrm{NaBH}_{4}$ to produce nanoparticle nuclei, and then growing these in a solution containing trisodium citrate and $\mathrm{H}_{2} \mathrm{O}_{2}$ [13] which produces a solution with a vibrant blue colour. Samples for the STEM were prepared by simply dipping a $30 \mathrm{~nm}$ thick $\mathrm{Si}_{3} \mathrm{~N}_{4}$ 
membrane substrate into the solution. The sample was given an air-ion plasma clean of approximately 20 minutes to reduce carbon contamination before insertion in the microscope. Periodically, before measurements, the sample area was subjected to a beam flooding procedure in order to fix any residual carbon contamination in place.

The EELS measurements were performed using a JEOL-ARM 2000F STEM operated at $200 \mathrm{kV}$ and fitted with a Gatan GIF Quantum spectrometer. The extraction voltage was lowered in order to reduce the energy resolution to $\sim 0: 5 \mathrm{eV}$ (as measured by the full width half maximum (FWHM) of the zero loss peak (ZLP)). This also resulted in an approximate 10x decrease in emission current. The STEM was operated with aberration correction, giving a probe size of $\sim 0: 1 \mathrm{~nm}$ and $\sim 0.015 \mathrm{nA}$ of current (after reduction of the source extraction voltage). The convergence and collection angles of the beam were $\sim 24.9$ mrad and $\sim 8.8$ mrad respectively.

EEL spectrum images were captured using 20 acquisitions at each pixel with an acquisition time of 0.05 to $0.1 \mathrm{~s}$ each. The energy dispersion was set to $0.05 \mathrm{eV}$ per pixel and spatial drift correction during acquisition was applied. Full on-chip binning was used. Gatan's post-acquisition darkcurrent correction routine was applied and this improves the statistical accuracy via repeated and averaged dark current collection. The ZLP was removed using a power-law fitting below the lowest energy feature (the dipole resonance) with extrapolation to higher energies. Although this approach may mean that the relative intensities of the spectral features may not be quite correct, it avoids the introduction of spurious features that is inherent to more sophisticated ZLP extraction processes such as subtraction or deconvolution of a nominal ZLP. Principal component analysis (PCA) was performed followed by reconstruction with the first $\sim 12$ components in order to reduce random noise [37]. It was necessary to crop the energy scale down to a maximum of $\sim 8.5 \mathrm{eV}$ in order for the PCA analysis to be effective. Maps of the intensity distribution of the LSPR peaks were obtained by Gaussian fitting to the features of interest.

Calculations were performed using a Matlab implementation of BEM, the MNPBEM Toolbox and its EELS extension [35,36]. The triangle parameters were determined from electron microscopy images of the particles and are given in Table 1 below. $L_{1}$ is the length of the triangle without any truncation of the tip, $r$ is the radius of curvature for the tip truncation and $z$ is the thickness of the triangle. The parameter $c_{z}$ determines the curvature of the edge of the triangle, $c_{z}=0$ corresponds to flat edges and $c_{z}=1$ corresponds to a hemispherical shape. The thickness of the substrate is $h$. The substrate thickness was set to the nominal value of $30 \mathrm{~nm}$. The triangle thickness and edge curvature were not measured for the particular triangles studied here but separate TEM studies of the solution indicated a narrow distribution of these values for nanoparticle solution and the values used here are expected to be representative of the actual value.

Table 1. Geometric parameters used in the BEM simulations.

\begin{tabular}{lccccc}
\hline & $L_{1}$ & $r$ & $z$ & $c_{z}$ & $h$ \\
\hline Triangle 1 & 110 & 15 & 6 & 0.85 & 30 \\
Triangle 2 & 170 & 16 & 6 & 0.85 & 30 \\
\hline
\end{tabular}

If the particle has a curved edge profile at the interface near the substrate, the two separate surfaces are in close proximity and artefacts are produced in the calculations. Therefore, at this interface the edge of the triangle was not curved. We have separately studied the effects of edge curvature on optical responses and have found the influence is small. Therefore, this approximation is unlikely to have a significant effect on the results presented here. A number of other parameters are used to set the size of the elements in the surface mesh used in the BEM and these were determined through convergence testing. 
The dielectric function for Ag was taken from Palik [38] and the dielectric function for the substrate was taken as a constant of 3.2. The dielectric constant for $\mathrm{Si}_{3} \mathrm{~N}_{4}$ depends on stoichiometry, which is not known for these substrates. The value only influences the energy position of spectral features, not their qualitative behaviour. The value chosen is within the known range for $\mathrm{Si}_{3} \mathrm{~N}_{4}$ and gave the best quantitative agreement for spectral positions.

\section{Results and Discussion}

EELS spectrum images were obtained from regions that contained nine triangular nanoplates altogether and these had a range of tip-to-tip edge lengths from 50 to $200 \mathrm{~nm}$. As has been previously observed, the main LSPR resonances decreased in energy as the triangle size increased [11]. Examples from two typical triangular nanoplates, having the geometry described by Table 1, are given here. Figs. 1 and 3 show the experimental and simulated spectra at the electron beam positions as indicated in the inset. Figs. 2 and 4 show experimental and calculated maps of the intensity of the LSPR excitation peaks as a function of beam position.

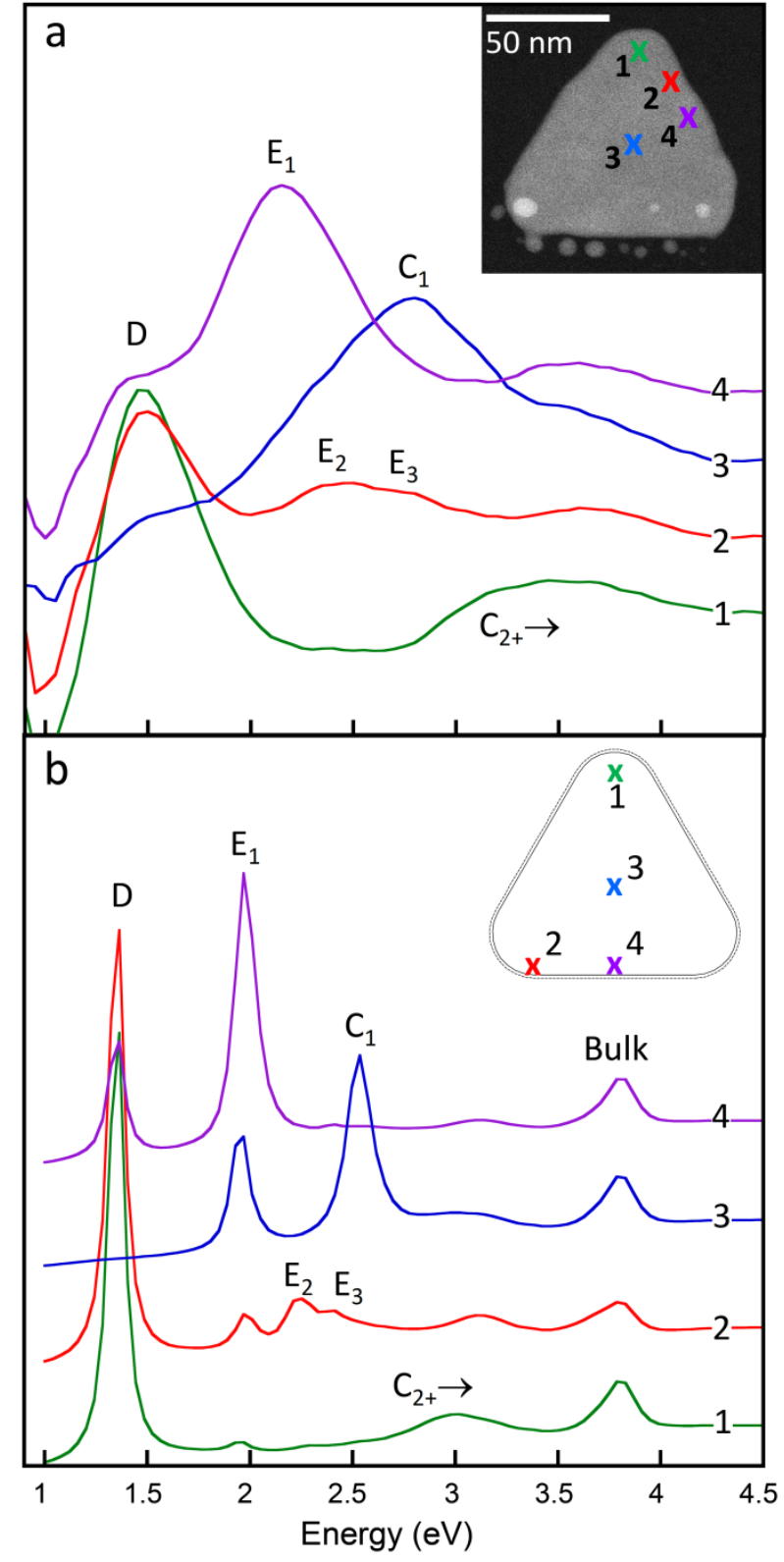

Fig. 1. (a) Experimental and (b) calculated EEL spectra for the electron beam positions as indicated by the respective insets, for a $\mathrm{Ag}$ triangular nanoplate having a side length (before truncation) of $110 \mathrm{~nm}$. a
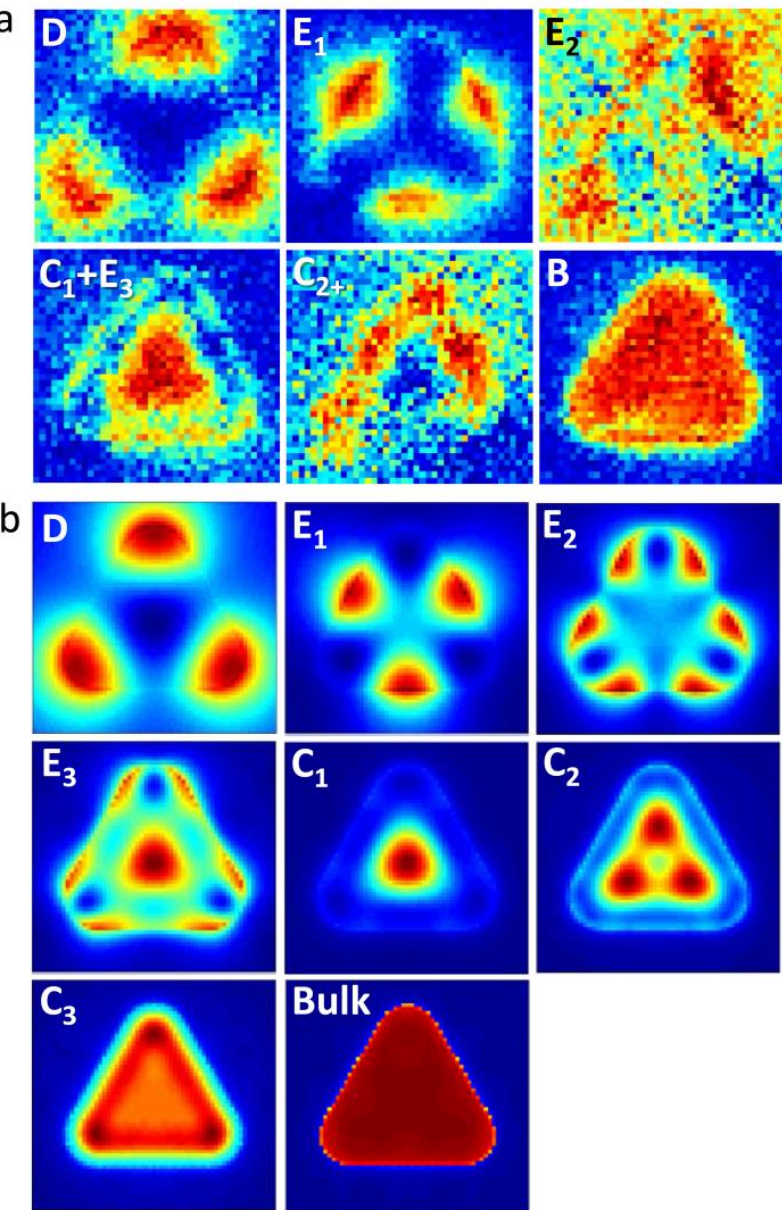

Fig. 2. (a) Experimental and (b) calculated EEL plasmon excitation maps from a Ag triangular nanoplate having a side length (before truncation) of $110 \mathrm{~nm}$. The maps have been generated for the spectral features as labelled in Fig. 1. 


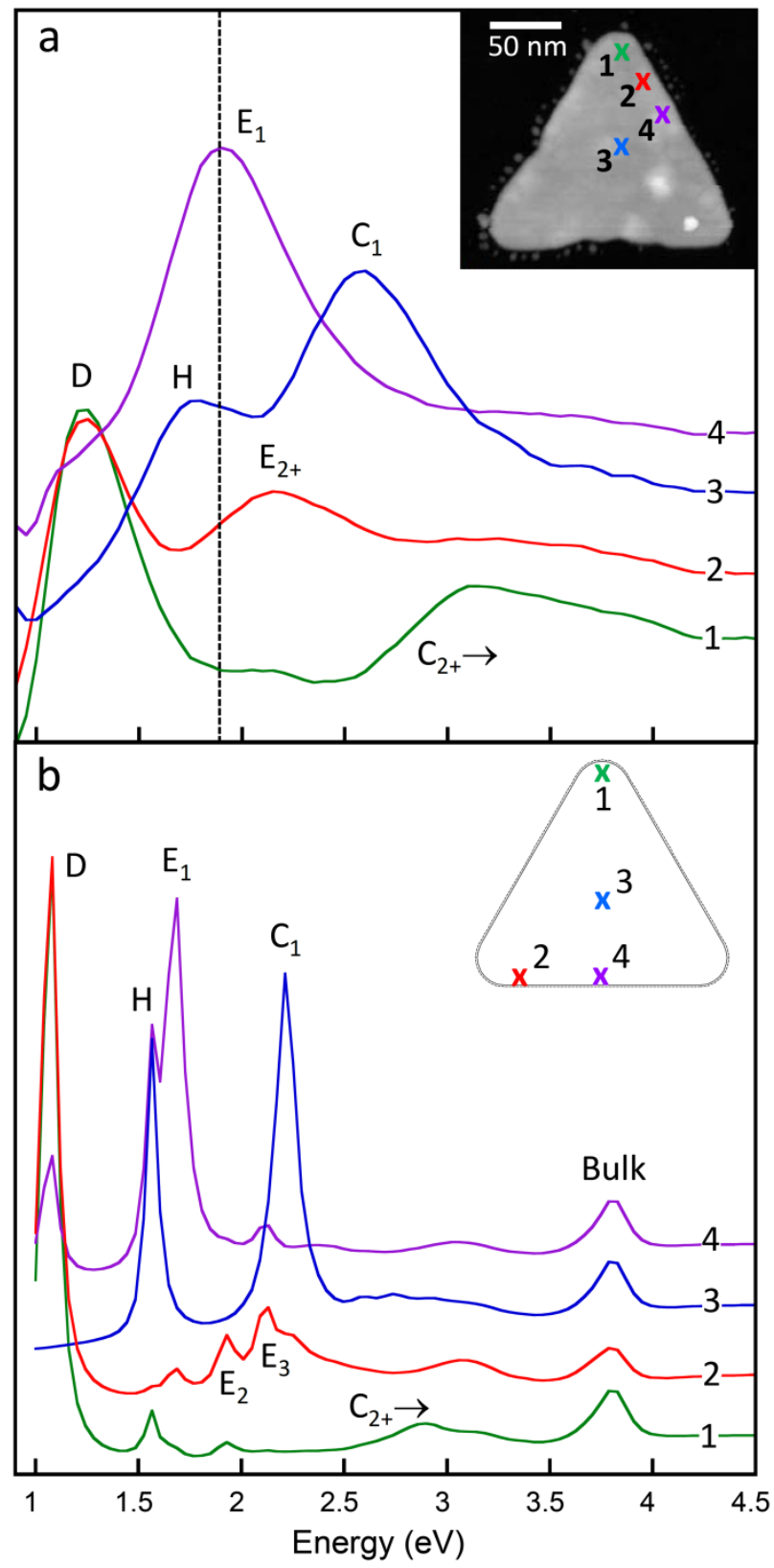

Fig. 3. (a) Experimental and (b) calculated EEL spectra for the electron beam positions as indicated by the respective insets, for a $\mathrm{Ag}$ triangular nanoplate having a side length (before truncation) of $170 \mathrm{~nm}$.
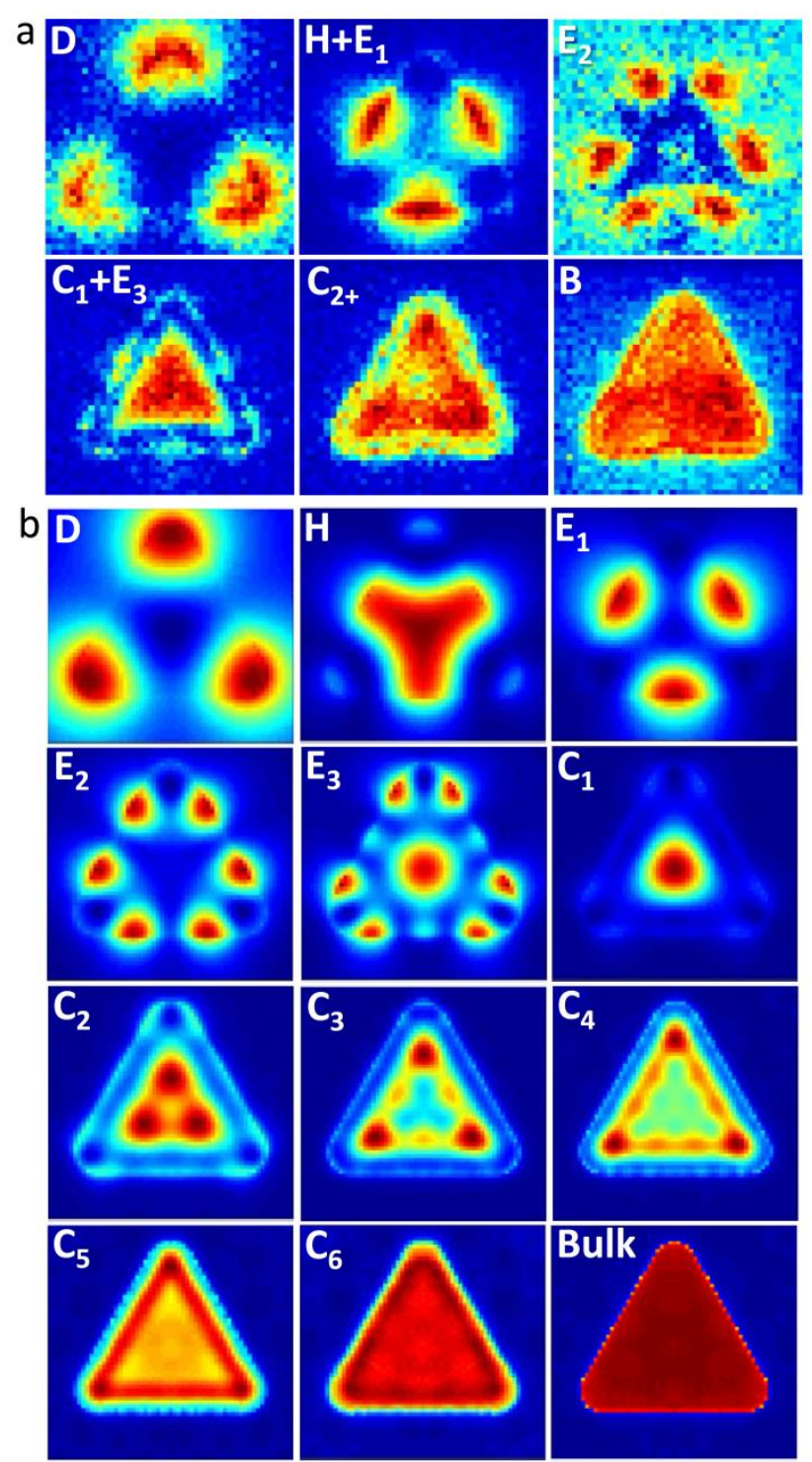

Fig. 4. (a) Experimental and (b) calculated EEL plasmon excitation maps from a Ag triangular nanoplate having a side length (before truncation) of $170 \mathrm{~nm}$. The maps have been generated for the spectral features as labelled in Fig. 3.

A number of distinct plasmon modes are observed in the triangular nanoplates. The lowest energy mode is the in-plane dipole mode (D) which is excited by an electron beam located at the tips of the triangles and this is also the dominant mode in optical extinction spectra from these nanoparticles. When the electron beam is located in the centre of the edge of the triangle the lowest order edge mode $\left(E_{1}\right)$ is excited and this mode has previously been identified as corresponding to the optically excited quadrupole mode. Higher order edge modes $\left(\mathrm{E}_{2}, \mathrm{E}_{3}\right)$ and an optically dark centre mode $\left(\mathrm{C}_{1}\right)$ are also observed which is consistent with previous measurements [11,27-33]. 
However, new modes are also observed when the electron beam is located on the particle. These are higher order centre modes $\left(\mathrm{C}_{2}, \mathrm{C}_{3}\right.$ and so on) which are seen in both particles. Furthermore, in the larger particle (Figs. 3, 4) another mode $(\mathrm{H})$ with three-fold symmetry is excited and this appears to be a hybrid between the edge mode $\left(\mathrm{E}_{1}\right)$ and the centre mode $\left(\mathrm{C}_{1}\right)$. This $\mathrm{H}$ mode was only observed in the larger particles. However, its appearance is believed to be connected to the triangular geometry rather than size. Smaller particles generally have more rounded tips and this breaks the symmetry conditions for the formation of the hybrid mode.

The centre modes of different orders are similar to the "breathing modes" previously observed in nanodisks $[32,39,40]$ but altered by the triangular geometry. They can be thought of as propagating surface plasmons that are instead confined to the triangular shape. They are entirely analogous to the laser modes in triangular cavities described by Chang [41]. Wavevectors derived from description of Chang were used with a calculated dispersion relation for propagating surface plasmons on thin Ag films [42] and gave the energies observed for these higher order centre modes, thus verifying this interpretation. These modes correspond to modes with a symmetric charge distribution on the two faces. There is evidence in the calculations that the weaker, higher energy anti-symmetric modes are also present, however these cannot be distinguished in the experimental spectra.

There is a small quantitative discrepancy between the experimental and calculated mode energies. This is not surprising given the assumptions made about the substrate (dielectric function and thickness) and the assumptions about the thickness and geometry of the particle. The particles do not show a perfect triangular shape and their thickness is likely to be slightly different from the value used in the calculations. There is also evidence in the images for the early onset of the corrosion process, perhaps accelerated by the plasma cleaning. Any residual carbon contamination is also not accounted for in the calculations.

The limited experimental energy resolution means that there is considerable overlap between the different modes. For the smaller particle, although there is a distinct shift upwards in the energy of the edge mode as the beam moves from the centre of the edge towards the tip, it was not possible to reliably extract a map of the higher order edge modes $\left(E_{2}, E_{3}\right)$. In the larger particle $E_{2}$ can be mapped because the peaks become more separated in energy, but there remains an overlap between $\mathrm{E}_{3}$ and $\mathrm{C}_{1}$. Similarly, the higher order centre modes $\left(\mathrm{C}_{2+}\right)$ cannot be individually distinguished and extracted in the experimental data, but their presence is clear in the spectra where it is observed that the centre mode moves up in energy as the beam moves from the centre of the particle towards the edge. The $\mathrm{H}$ mode cannot be separated in the experimental data but there is a clear shift down in energy as revealed by the dotted vertical line in Fig. 3a. Despite the limitations in experimental energy resolution, the new modes revealed by the BEM calculations are clearly supported by the experimental data.

\section{Conclusion}

It has been shown that a variety edge and internal (centre) plasmonic modes can be excited on $\mathrm{Ag}$ triangular nanoplates. These modes have been observed experimentally using an aberration corrected JEOL-ARM microscope and theoretically verified with calculations using the BEM. A novel centre-edge hybrid mode occurs in triangles with sharp tips. Higher order internal modes are also observed for the first time and these can be interpreted as planar surface modes that are confined to the triangular shape. These higher order internal modes are entirely analogous to triangular laser cavity modes and have wave-vectors that can be described in the same manner. The internal modes are expected to be optically dark under conventional illumination but could be revealed using oblique optical illumination [43] or a nano-antennae [26]. 


\section{References}

1. Brongersma ML (2015) Introductory lecture: nanoplasmonics. Faraday Discussions 178:9-36

2. Goncalves MR (2014) Plasmonic nanoparticles: fabrication, simulation and experiments. J Phys

D: Appl Phys 47:213001

3. Lindquist NC, Nagpal P, McPeak KM, Norris DJ, Oh S-H (2012) Engineering metallic nanostructures for plasmonics and nanophotonics. Rep Prog Phys 75:036501

4. Rycenga M, Cobley CM, Zeng J, Li W, Moran CH, Zhang Q, Qin D, Xia Y (2011) Controlling the synthesis and assembly of silver nanostructures for plamonic applications. Chemical Reviews 111:3699-3712

5. Schuller J, Barnard ES, Cai W, Jun YC, White JS, Brongersma ML (2010) Plasmonics for extreme light concentration and manipulation. Nature Materials 9:193-204

6. Millstone JE, Hurst SJ, Métraux G, Cutler JI, Mirkin CA (2009) Colloidal gold and silver triangular nanoprisms. Small 5 (6):646-664

7. Aherne D, Ledwith DM, Gara M, Kelly JM (2008) Optical properties and growth aspects of silver nanoprisms produced by a highly reproducible and rapid synthesis at room temperature. Adv Funct Mater 18:2005-2016

8. Charles DE, Aherne D, Gara M, Ledwith DM, Gun'ko YK, Kelly JM, Blau WJ, Brennan-Fournet ME (2010) Versatile solution phase triangular silver nanoplates for highly sensitive plasmon resonance sensing. ACS Nano 4 (1):55-64

9. Jensen TR, Duval Malinsky M, Haynes CL, Van Duyne RP (2000) Nanosphere lithography: tunable localized surface plasmon resonance spectra of silver nanoparticles. J Phys Chem B 104:10549-10556

10. Mock JJ, Barbic M, Smith DR, Schultz DA, Schultz S (2002) Shape effects in plasmon resonance of individual colloidal silver nanoparticles. J Chem Phys 116 (15):6755-6759

11. Nelayah J, Kociak M, Stephan O, Geuquey N, Henrard L, Garcia de Abajo FJ, Pastoriza-Santos I, Liz-Marzan LM, Colliex C (2010) Two-dimensional quasistatic stationary short range surface plasmons in flat nanoprisms. Nano Letters 10:902-907

12. Shuford KL, Ratner MA, Schatz GC (2005) Multipolar excitation in triangular nanoprisms. J Chem Phys 123:114713

13. Tsuji M, Gomi S, Maeda Y, Matsunaga M, Hikino S, Uto K, Tsuji T, Kawazumi H (2012) Rapid transformation from spherical nanoparticles, nanorods, cubes, or bipyramids to triangular prisms of silver with PVP, citrate and $\mathrm{H}_{2} \mathrm{O}_{2}$. Langmuir 28:8845-8861

14. Haes AJ, Zou S, Schatz GC, Van Duyne RP (2004) Nanoscale optical biosensor" short range distance dependence of the localized surface plasmon resonance of noble metal nanoparticles. $\mathrm{J}$ Phys Chem B 108:6961-6968

15. Lu L, Kobayashi A, Tawa K, Ozaki Y (2006) Silver nanoplates with special shapes: controlled synthesis and their surface palsmon resonance and surface-enhanced Raman scattering properties. Chem Mater 18:4894-4901

16. Ledwith DM, Whelan AM, Kelly JM (2007) A rapid, straight-forward method for controlling the morphology of stable silver nanoparticles. J Mater Chem 17:2459-2464

17. Zeng J, Roberts S, Xia Y (2010) Nanocrystal -based time-temperature indicators. Chem Eur J 16:12559-12563

18. Charles DE, Gara M, Aherne D, Ledwith DM, Kelly JM, Blau WJ, Brennan-Fournet ME (2011) Scaling of surface plasmons resonances in triangular silver nanoplate sols for enhanced refractive index sensing. Plasmonics 6:351-362

19. Le Beulze A, Duguet E, Mornet S, Majimel J, Trèguer-Delapierre M, Ravaine S, Florea I, Ersen $\mathrm{O}$ (2014) New insights into the side-face structure, growth aspects and reactivity of $\mathrm{Ag}_{\mathrm{n}}$ nanoprisms. Langmuir 30:1424-1434

20. Chen S, Carroll DL (2002) Synthesis and characterization of truncated triangular silver nanoplates. Nano Letters 2 (9):1003-1007

21. Jin R, Cao YW, Mirkin CA, Kelly KL, Schatz GC, Zheng JG (2001) Photinduced conversion of silver nanospheres to nanoprisms. Science 294:1901-1903 
22. Sherry LJ, Chang SH, Schatz GC, Van Duyne RP (2005) Localized surface plasmon resonance spectroscopy of single silver nanocubes. Nano Letters 5 (10):2034-2038

23. Munechika K, Smith JM, Chen T, Ginger DS (2007) Plasmon line widths of single silver nanoprisms as a function of particle size and plasmon peak position. J Phys Chem C 111:1890618911

24. Blaber MG, Henry AI, Bingham JM, Schatz GC, Van Duyne RP (2012) LSPR imaging of silver triangular nanoprisms: correlating scattering with structure using electrodynamics for plasmon lifetime analysis. J Phys Chem C 116:393-403

25. Kelly RL, Coronado E, Zhao LL, Schatz GC (2003) The optical properties of metal nanoparticles: the influence of size shape and dielectric environment. J Phys Chem B 107:668-677 26. Fletcher G, Arnold MD, Pedersen T, Keast VJ, Cortie MB (2015) Multipolar and dark-mode plasmon resonanced on drilled silver nano-triangles. Optics Express 23 (4):18002-18013

27. Gu L, Sigle W, Koch CT, Ögüt B, Van Aken PA, Talebi N, Vogelgesang R, Mu J, Wen X, Mao J (2011) Resonant wedge-plasmon modes in single crystalline gold nanoplatelets. Phys Rev B 83:195433

28. Koh AL, Fernandez-Dominguez AI, McComb DW, Maier SA, Yang JKW (2011) Highresolution mapping of electron-beam-excited plasmon modes in lithographically defined gold nanostructures. Nano Letters 11:1323-1330

29. Losquin A, Zagonel LF, Myroshnychenko V, Rodriquez-Gonzalez B, Tence M, Scarabelli L, Forstner J, Liz-Marzan LM, Garcia de Abajo FJ, Stephan O, Kociak M (2015) Unveling nanometer scale extinction and scattering phenomena through combines electron energy loss spectroscopy and cathodoluminescence measurements. Nano Letters 15:1229

30. Nelayah J, Gu L, Sigle W, Koch CT, Pastoriza-Santos I, Liz-Marzan LM, Van Aken PA (2009) Direct imaging of surface plasmon resonances on single triangular silver nanoprisms at optical wavelength using low-loss EFTEM imaging. Optics Letters 34 (7):1003-1005

31. Nelayah J, Kociak M, Stéphan O, García de Abajo FJ, Tencé M, Henrard L, Taverna D, Pastoriza-Santos I, Liz-Marzán LM, Colliex C (2007) Mapping Surface plasmons on a single metallic nanoparticle. Nature Physics 3:348-353

32. Schmidt FP, Ditlbacher H, Hofer F, Krenn JR, Hohenester U (2014) Morphing a plasmonic nanodisk into a nanotriangle. Nano Letters 14:4810-4815

33. Talebi N, Sigle W, Vogelgesang R, Koch CT, Fernandez-Lopez C, Liz-Marzan LM, Ogut B, Rohm M, van Aken PA (2012) Breaking the mode degeneracy of surface plasmon resonances in a triangular system. Langmuir 28:8867-8873

34. Garcia de Abajo FJ, Howie A (2002) Retarded field calculation of electron energy loss in inhomogeneous dielectrics. Phys Rev B 65:115418

35. Hohenester U (2014) Simulating electron energy loss spectroscopy with the MNPBEM toolbox. Computer Physics Communications 185 (1177-1187)

36. Hohenester U, Trugler A (2012) MNPBEM - A Matlab toolbox for the simulation of plasmonic nanoparticles. Computer Physics Communications 183:370-381

37. Watanabe M, Okunishi E, Ishizu K (2009) Analysis of Spectrum-Imaging Datasets in Atomic-Resolution Electron Microscopy. Microscopy and Analysis 23 Nov.:5-7

38. Palik ED (1998) Handbook of optical constants of solids. Academic Press, 39. Schmidt FP, Ditlbacher H, Hohenester U, Hohenau A, Hofer F, Krenn JR (2012) Dark plasmonic breathing modes in silver nanodisks. Nano Letters 12:5780-5783 40. Schmidt FP, Ditlbacher H, Hohenester U, Hohenau A, Hofer F, Krenn JR (2013) Universal disperson of surface plasmon nanostructures. Nature Communications 5:3604

41. Chang HC, Kioseoglou G, Lee EH, Haetty J, Na MH, Xuan Y, Luo H, Petrou A, Cartwright AN (2000) Lasing modes in equilateral triangular laser cavities. Phys Rev A 62:013816

42. Bolton JPR, Chen M (1995) Electron energy loss in multilayered slabs. Ultramicroscopy 60

(247-263)

43. Krug MK, Reisecker M, Hohenau A, Ditlbacher H, Trugler A, Hohenester U, Krenn JR (2014) Probing plasmonic breathing modes optically. Appl Phys Lett 105:171103 
